\title{
PAPILEDEMA UNILATERAL NA STNDROME DO PSEUDOTUMOR CEREBRAL
}

\author{
MARIO L. R. MONTRIRO * \\ WILLIAM F. HOYT ** \\ RICHARD K. IMES * \\ MICHAEL NARAHARA "**
}

Edema unilateral da papila óptica geralmente indica patologia ocular ou orbitária. Há, contudo, exceçōes a essa regra geral. Edema de disco decorrente de aumento da pressāo intracraniana (papilederna) já foi relatado como achado unilateral em pacientes com tumor cerebral 1,10 e, mais recentemente, com a sindrome do pseudotumor cerebral $8, \theta, 11$.

Neste trabalho, documentamos a ocorrência e discutimos os possiveis mecanismos patogenéticos de papiledema unilateral em 6 pacientes com aumento da pressăo intracraniana causada pela sindrome do pseudotumor cerebral.

\section{CAEUISTICA}

On achados clinicos e exames complementares de 6 paclentes com paeudotumor cerebral e papiledema unllateral foram revistos. Todos of pacienteg toram submetldos a avalfacoes neurologica e oftalmolótica no Departamento de Neurologia e Unidade de Neuro-oftalmologia da Unjersidade da California em sto Franciseo. Investjgactea neurorradiológleas consiatiram em tomografia computadorizada (TC) do córebro em 5 pacientes e pneumeneetalografia agsociada a tomografia do cranlo em um paciente (caso 3). A puncto lombar revelou sumento da preseăo intracraniana, estabelecendo o diagnóstico de peeudotumor cerebral em cada um dos pacientes. Todos os pacientes cram do bexo femintno, com ldxde entre 24 e 69 anos.

\section{RFIULTADOS}

Na tabela 1 gato resumidos of achados noe 6 pacientea. Cinco deles se quelzaram de obscurecimentoa transitórios da visăo no olho com paplledema, tendo a duraço dt

Trabalho realizado na Unidede de Neuro-oftalmologia, Departamentos de Neurocirurgla, Neurologla $\theta$ Oftalmologia, Universidade da Cajifornia em Sto Franciaco, URA: * Fellow em Neuro-oftajmologla; ** Profeseor, Departamentos de Neurocirurgle, Neurologla e Oftalmologis; "* Fotógrafo, Departamento de Oftalmologia Enderecto atual do Dr. M.L.R. Monteiro: Divipto de clinica Oftalmológlea do Hospital da Clínicas da Faculdade de Medielna da Univerbidade de sto Pauto (Gervięo do Prot. Jorge A.F. Calde(ra). 
apenes alguns segundos e geralmente preciptados por alteraçoes da posicta corporal, tais como o ato de se leventar ou de se curvar. Tres pactentes tinham também cefalés frontal o um era assintomatico. Todos tinham aculdade visual normal a aumento da mancha cega no olho com papiledema $O$ exame neurológico fol eernpre normal. A presab̆o intracraniang oscilou entre 260 e $410 \mathrm{mmH}_{2} O$. Dols pacientes tinham evidencig radiografica de sindrome da sela vazla percial mas auas avaljacoes endocrinologicas foram normals. Fhe dois casos a TC demonstrou aumento de imagem dos nervos opticos e, em um deles, tambêm tamanho pequeno doa ventrículos.

\begin{tabular}{|c|c|c|c|}
\hline Caso & $\begin{array}{l}\text { Achados } \\
\text { clinicos }\end{array}$ & $\begin{array}{l}\text { Achados } \\
\text { neurorradiologlcos }\end{array}$ & $\begin{array}{l}\text { Presagto } \\
\text { Intracraniana } \\
\left(\mathrm{mm} \text { de } \mathrm{H}_{2} \mathrm{O}\right)\end{array}$ \\
\hline 1 & $\begin{array}{l}\text { Cefaléla frontal. obscura- } \\
\text { cóes trangitorias da viado. } \\
\text { obegldade }\end{array}$ & $\begin{array}{l}\text { Aumento de imsgem dos nervos } \\
\text { opticos }\end{array}$ & 280 \\
\hline 2 & $\begin{array}{l}\text { Cefaléla trontal, obscura- } \\
\text { çes transitórias da viako, } \\
\text { obesidade }\end{array}$ & Normal & 270 \\
\hline 3 & $\begin{array}{l}\text { Obscuraçoes trangitórias de } \\
\text { visÁo }\end{array}$ & Sindrome da pela vazia parcial & 240 \\
\hline 4 & $\begin{array}{l}\text { Cefaléla frontal, obacura- } \\
\text { çoes transitórias da viafo, } \\
\text { obesidade }\end{array}$ & $\begin{array}{l}\text { Pequenos ventriculos, eumento } \\
\text { da imagem dos nervos opticos }\end{array}$ & 300 \\
\hline $\mathbf{5}$ & Oberidade & Sindrome da sela vazia parclal & 250 \\
\hline 6 & $\begin{array}{l}\text { Obscuragúes transitórias da } \\
\text { Fleăo, obesldade }\end{array}$ & Normal & 410 \\
\hline
\end{tabular}

Tabela 1 - Rearma dos achodos am 6 pactentes com a sfidrome do pseudotumor cerebral e payiledema unilateral.

O sppecto oftalmoscóploo da papllas opticas a spresentado nas flgurag 1 e 2. Tres pacientes tinham papiledems efritamento unilateral (FIg. 1) enquanto que os outros tinham mínimos sinsia de edema de aiseo no olho contralateral (Fig. 2), Itm todos oB casos, o olho afetado apresentava papiledems totslmente desenvolvido com as aeguintes caracteriaticas: borramento das margens em 360 graus do diaco optico; preenchimento da escavaç̆ friologica, obscurecimento dos vasos do disco óptico, elevaço de 3 a 4 dioptrias. Havia digcreto ingurgitamento venoso e augencia de pulasço nas veias. Näo havla exaudatos ou hemorraglas. 


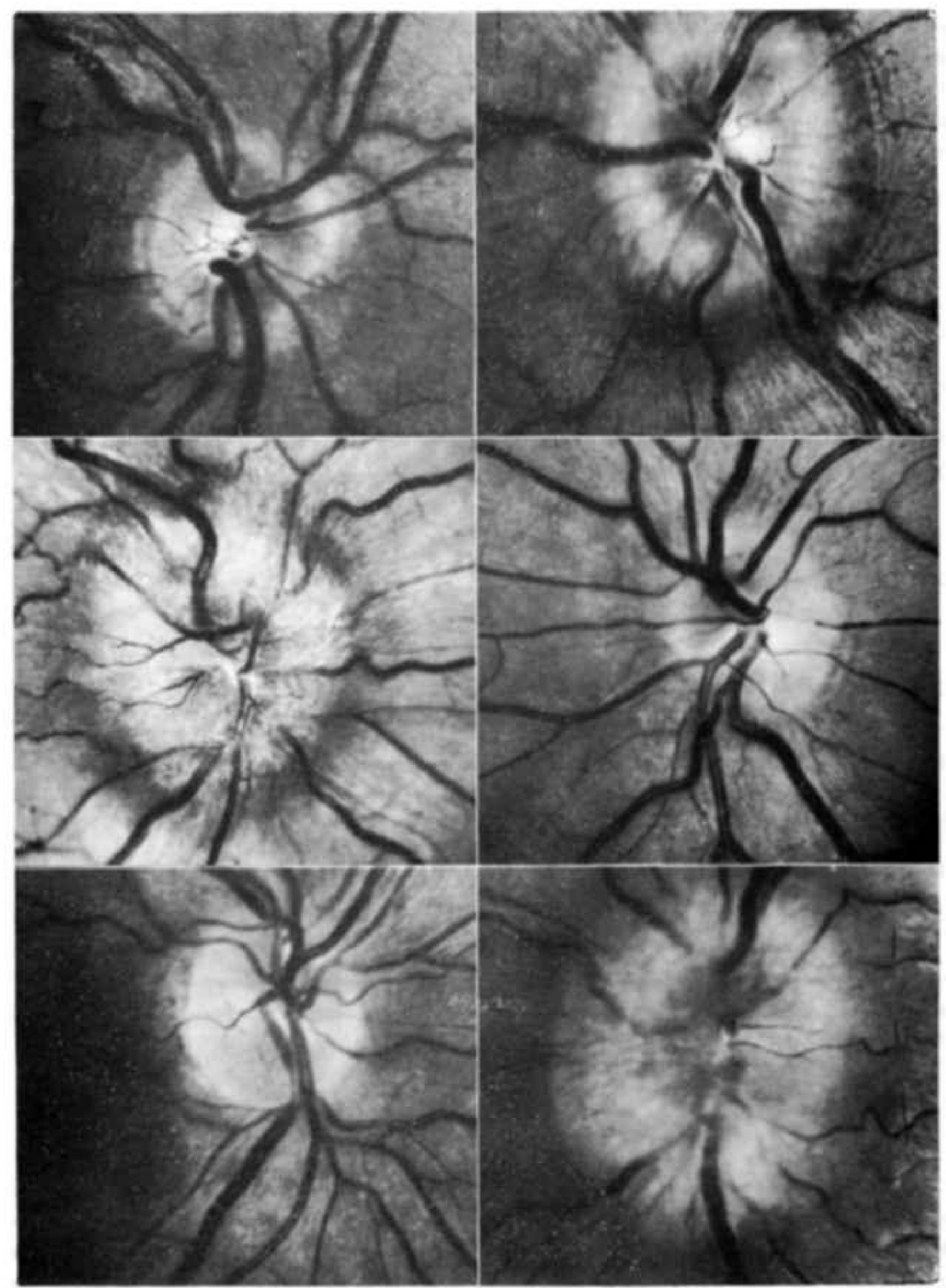

Fig. 1 - Papiledema unilateral em tres pacientes com pseudotumor cerebral. Acima, no meio e abaixo, os dois olhos dos casos 1,2 e 8 , respectivamente. A direita, olho direito (OD); a esquerda, olho esquerdo (OE). 


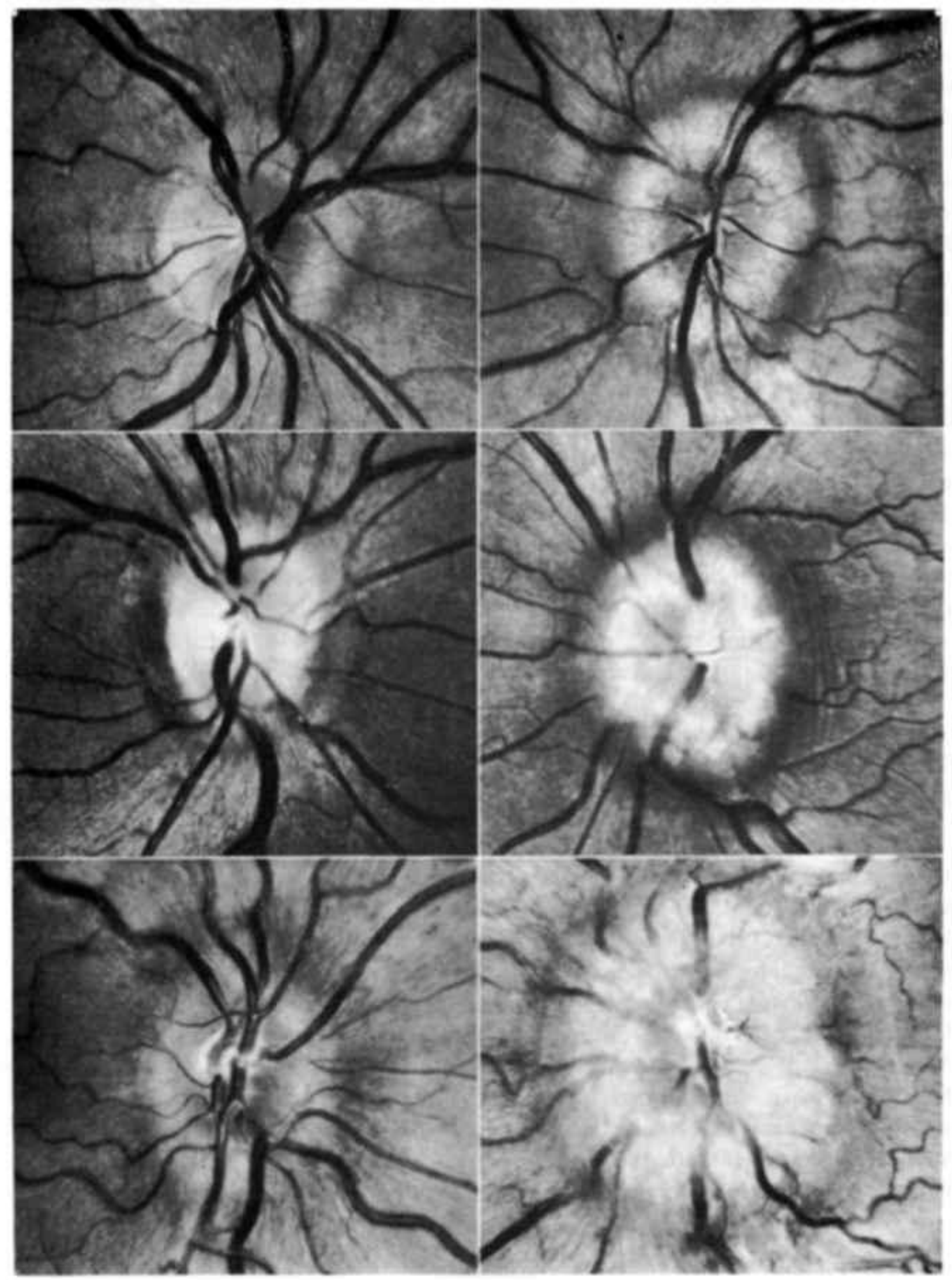

Fig. 2 - Papiledema assimétrico nos casos 4, 5 e 6. A direita OD; a esquerda OE. 


\section{COMENTARIOS}

O achado de papileỏema unilateral é raro na sindrome do pseudotumor cerebral. Quando isto ocorre, pode haver confusåo diagnóstica com patologias oculares ou orbitárias, uma vez que tais pacientes em geral possuem exames neurológicos e neurorradiológicos normais. Em nossos casos, problemas oftalmológicos locais puderam ser clinicamente excluidos. Todos os pacientes tinham pressāo intracraniana elevada com análise do líquido cefalorraqueano (LCR) normal, ausência de sinais neurológicos e ventrículos pequenos ou normais nas investigą̧̃es neurorradiológicas, preenchendo portanto os critérios para o diagnóstico da síndrome do pseudotumor cerebral s. Os achados associados de obesidade e sindrome da sela vazia que ocorreram em alguns dos nossos pacientes, já foram amplamente descritos no pseudotumor cerebral 2,0 .

Papiledema é em geral bilateral e com intensidade semelhante nos dois olhos ${ }^{7}$. Hayreh ${ }^{3}$ demonstrou em macacos que um aumento da pressāo do fluído cerebrospinal no espaço subaracnóideo perióptico, é pré-requisito necessário ao desenvolvimento de papiledema. Isto leva à estase do fluxo axoplasmático e edema das fibras nervosas anteriores à lamina crivosa. Alteraçōes vasculares retinianas se desenvolvem secundariamente 4,12 , A ocorrência de edema unilateral de disco óptico em pacientes com pressão intracraniana elevada pode ser causada por fatores que impedem a transmissão da pressão do LCR através da bainha do nervo óptico de um dos lados. Isto pode ser um defeito congểnito, pós-inflamatório, ou causado por compressäo extrínseca sobre a bainha ". Um segundo mecanismo para a explicaçăo do papiledema unilateral é baseado na ocorrência de alteraçōes Jocais na cabeça do nervo óptico, tal como ocorre nos discos congenitamente displásticos ou nas atrofias ópticas $\sigma$. Essas alteraçōes impedem o desenvolvimento do edema de disco, a despeito de uma transmissăo normal da pressão intracraniana através do nervo óptico.

Kirkham et al. b, sugeriram que o papiledema unilateral em pacientes com pseudotumor cerebral era decorrente da presença de anomalia na bainha do nervo óptico, a qual impediria a transmissão da pressão do LCR para a cabeça do nervo óptico. No entanto, Muci-Mendoza et al, 9 relataram dois casos de papiledema unilateral em pseudotumor cerebral, nos quais a TC revelou aumento bilateral da imagem dos nervos ópticos na órbita indicando, portanto, patência do espaço subaracnóideo perióptico, mesmo no lado em que năo havia papiledema. Parece, portanto, mais provável que os fatores anatómicos e fisiológicos que determinam se o disco óptico irá ou não desenvolver edema, em pacientes com pressão intractaniana elevada, devem operar na porçăo distal do nervo óptico 8 . Năo se sabe, no entanto, se este seria o único mecanismo para o desenvolvimento de papiledema unilateral na sindrome do pseudotumor cerebral.

\section{REPSUMO}

Sazo apresentadas as fotografias de fundo de olho de 6 mulheres com papiledema unilateral e a síndrome do pseudotumor cerebral. Achados associados foram: obesidade em 5 e a síndrome da sela vazia parcial em 2 . Sáo discutidas 
as possiveis explicaçöes para o desenvolvimento de papiledema unilateral nesses pacientes e que favorecem um mecanismo presente na porçăo distal dos nervos opticos.

\section{BUMMARY}

\section{Unilateral papilledema in pseudotumor cerebri.}

The appearance of unilateral papilledema in the fundus photos of 6 women with pseudotumor cerebri is presented. Associated findings included obesity in 5 and a partially empty sella in 2 . The possible explanations for the development of unilateral papilledema in pseudotumor cerebri are reviewed and favor a mechanism that is present at the distal end of the optic nerves.

\section{REFFERONCLAB}

1. BRUNTse, E. - Unllateral paplliedema in neurosurgieal patients, Acto Ophthalmol. $48: 759,1970$.

2. FOLfry. K.M. \& POSNiR, J.B. - Does peeudomotor cerebr chuBe the empty sella Eyndrome? Neurology (Minneapolia) 25:565, 1975.

3. HAYREH, 5.s. - Pathogeneelp of oedema of the optic diac (papilloedema). A preliminary report. Brit. J. Ophthalmol. 48:592, 1964.

4. HAYREH, 6.8. - Optic disc edema in raibed intracranial presaure. V. Pathogenesia. Arch, Ophthalmol. S5:1563, 1977.

5. JOFNGTON, I. \& PATERBON, A. - Benlgn Intracranfel hypertengion: I. Diagnople und prognowis. Brain $9: 289,1974$.

B. KIRKHAM, T.H.; BANDKhR, M.D. \& SAPP, G.A - Unilateral papilloederns in benign Intracranial hypertenaion. Can. J. Ophthalmol. 8:533, 1973.

7. MILTFR, N.R. - Walsh and Hoyt's Clinleal Neuro-Ophthalmology. zrd. 4. Willems A Wiksins, Raltimore, 1982, pg. 187.

8. MUCI-MENDOZA, R. - Unilateral and agymmetric optic disc ewelling with intracranial abnormaltties. Amer. J. Ophthalmol, $\$: 858$, 1984.

9. MUCI-MRNDOZA, R. ARRUGA, J. HOYT, W.F. - Distension blateral del eqpacio subaracnoldeo pordoptloo en el pseudotumor cerebral con peplledemg umilateral. Bu demonstración a traves de la tomoxrafla computorisada de la orblta. Rev. Neurol. (Barcelona) 39:1t, 1981.

10. BEPWICK, L,A. \& BURDE, R.M. - Unilateral and asymmetrle optic dise gwelling intracranial abnormalities. Amer. J. Ophthalmol 98:484, 1989.

11. BHHR, N.A.; WIRTSCHAFTER, J.; SHAPIRO, B.K.; BEE, C. \& GHAPIRO, I. Unllateral paptledema in abenigns intracranial hypertension (paetdotumor cerebrl). J. amer. med. AsBoc. 2\%0:2846, 1983.

12. T\&O, M.O. \& HAYREH. 8.8. - Optle dise edema in rafsed Intracraniai greppure. IV. Axoplesmic tranaport In experimental papljedema. Arch. Ophthalmol, 86:1458. 197.

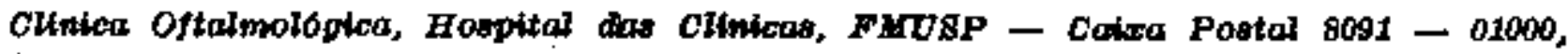
SAo Pawio, BP - Branl. 